\title{
CONGESTION CONTROL USING FUZZY BASED LSPS IN MULTIPROTOCOL LABEL SWITCHING NETWORKS
}

\author{
Anju Bhandari and V.P. Singh \\ Department of Computer Science and Engineering, Thapar University, Patiala, Punjab
}

\begin{abstract}
In this paper, we have proposed a fuzzy based decision making component for high volume traffic MPLS networks, by implementing Traffic Engineering, Quality of Service and Multipath routing. The approach explicitly proves to be successful in solving the issues and challenges pertaining to stability, scalability in high volume and dynamic traffic. Furthermore, it will work to handle congestion by higher link utilization and provides efficient rerouting of traffic along with fault tolerance in the network. In this research work, fuzzy calculations are done for fixing the attributes of the MPLS label(s), which is put on particular packet representing the Forwarding Equivalence Class. Fuzzy controller consists of two sub fuzzy systems- Label Switched Path setup System (LsS) and Traffic Splitting System (TSS). The computation of dynamic status of Load and Delay is utilized by LsS to arrange the paths in preference order. The attained Link Capacity and Utilization Rate are employing by TSS for maintaining congestion free path. The impact of this is to facilitate, we have better decision making for splitting the traffic for different promising paths. This was apparent from the series of traffic scenarios. Observations are obtained using this realization.
\end{abstract}

\section{KEYWORDS}

Multi Protocol Label Switching, Label Switched Path, Fuzzy Mixed Metric, Quality of Service, Traffic Splitting, Traffic Regulator.

\section{INTRODUCTION}

Due to the characteristics like high speed traffic, rapid topological changes and repetitive discontinuities. It is difficult to design an efficient protocol for MPLS networks. The MPLS is basically works on the concept of Label Switched Router (LSR) and helps to control the flow of the traffic. In this research work, Fuzzy calculations are done for fixing the attributes of the MPLS label, which is put on particular packet representing the Forwarding Equivalence Class (FEC). Since, MPLS packets have a different Layer 2 encoding [1-3]. The receiving LSR is aware of the MPLS packet, based on the layer 2 encoding. The range of labels ranges from 0 all the way through (20^20-1). Leaving aside the reserved and future ranges, our fuzzy based labels (LsS_value, TSS_value) arrives at these crisp values to take decision on path routing. The impact of this is that, we have better decision making for splitting the traffic for different possible paths. This was apparent from the series of traffic scenarios. An observation is obtained using this implementation and portrays the results in section afterward.

In terms of speed and scalability, today MPLS network is managing video traffic and it works well in adversity attacks like denial-of-service (DDoS) [4] attacks. When the data volume is too high and does not follow a predictable pattern, it becomes very challenging. Although, traffic splitting is the only condition to maintain business continuity and various statistical methods, machine learning methods have been implemented to understand traffic patterns and to take traffic engineering decisions. But these methods suffer from issues like large overhead and shallow analysis of current traffic. The dynamic rule based Engines are best suited for taking Traffic Engineering (TE) decisions because they can be utilized for fine tuning the TE decision 
with low computation cost. The information collection of requirement characterization and the network state are very difficult tasks in such a highly dynamic networks, which is the motivation due to which researchers have used Fuzzy Logic (FL) for solving these problems. It is one of the key issues to provide end-to-end QoS guarantees in today's networks. However, determining a feasible route in the current scenario of traffic along with its constraint is difficult to model and it is even harder to build a solution that will keep the network congestion free. In this case, FL can be a really helpful. FL has been found in many applications of telecommunications networks. In $[13,14]$ many different fuzzy logic controllers were proposed for traffic management in ATM networks and also to provide QoS in Communication networks. Hence, an idea of applying FL on the networks is to get better traffic management is surveyed in the paper.

The paper is organized with following details. Section 2 gives short description of stack of MPLS protocols. The Section 3 and 4 describes the proposed algorithm to overcome the limitation of existing MPLS based protocols used for traffic engineering. Section 5 demonstrates and gives analytical view of the theory of simulation done to validate the proposed algorithm. Finally, discussion and conclusions are presented.

\section{RELATED WORK AND DISCUSSION}

In the traditional approach, all types of traffic packets are treated equally. This is acceptable for the application like e-mail and those having no specific requirements for latency or bandwidth, but it becomes unacceptable and very challenging for high-speed networks, such as the real time applications and mission critical applications e.g. IP telephony, gaming, hard Quality of Service (QoS) demanding services [5, 6]. One of the given solution is traffic management with MPLS protocol. Several heuristics and approximation algorithms have been proposed for this problem. But, most of them suffer from either excessive computational cost or low performance.

Algorithms [7-8] like Minimum Hop Algorithm (MHA), Widest Shortest Path algorithm (WSP) and Shortest Widest Path (SWP) are based on the concept of combination of Shortest Path and Traffic Engineering, but these are not able to give reliable traffic scenario of the network. Minimum Interference Routing Algorithm (MIRA) and New Minimum Interference Routing Algorithm (NewMIRA) are based on the combination of TE and QoSR constraints. MIRA works on the potential traffic of Ingress-Egress routers as a weight to calculate shortest path. It is based on the observation that usage of certain critical links must be avoided to some possible extent. The objective in MHA is to minimize resource usage. It does not permit the protection of certain ingress-egress pairs from having their available capacities being reduced too much by traffic traversing between other ingress-egress pairs. It takes these factors into account when determining the path for the current demand. It performs explicit routing of Label Switched Path (LSP) in MPLS networks. Fowler et al. [10] proposed and evaluated a method that selects alternative routes based on the minimum time delay between the Ingress and the Egress when a new traffic flow arrives. Wu Hsiao Hsu et al. [11] proposed load balancing approach in handling the high volume traffic problems. The load sensitive approach maximizes resource utilization, and leads to a high number of packets reaching the destination. [12] Introduced an approach that established alternate paths and switch the ongoing traffic on them before the existing paths are pre-empted. It causes a loss of network throughput due to delay occurred and selects alternative paths when links become overloaded to give superior performance over routing algorithms.

Fuzzy algorithms [13-31] are based on different fuzzy parameters to give desirable output in different networks. Authors [15] utilized FL to model the multiobjective routing problem in mobile Ad-hoc networks. Authors [16] proposed a method for building a fuzzy system capable of predicting the QoS performance of each queue in a DiffServ node. Authors [17] applied the abilities of FL to propose a technique for designing a fuzzy based traffic conditioner. Nahid Ebrahimi Majd [28] found that fuzzy approach has a lower average setup time for establishing a 
connection than the previous existing approach. He concludes parameters like propagation delay, bandwidth and loss probability to form Fuzzy Mixed Metric (FMM) and generates best path in MPLS networks. It gives more priority to loss probability metric than any other metrics. It studied that fuzzy approach enhances the throughput and overall network performance. FMM is more efficient and overall drop is minimum than Wang et al. and Single Mixed Metric (SMM). N.M.Din [29] introduced the FL predictor for the real time traffic for TE decisions. According to the research work, the fuzzy predictor is used to construct one output (utilization) from two fuzzy inputs (average rate, available bandwidth). As per the claims of author the network performance increases, so that efficiency. There is a necessity to increase the control and to provide intelligence to the network. Sergio Gramajo et al. [30] introduced traffic prioritization which identifies traffic and then paths are allocated to them. The demand of this technique is increasing day by day as it is very useful in critical network services. But, even then these are not able to fully utilize the resources and lead to over and under utilization of the routes. As per this paper, authors are advised to combine the features of TE, Quality of service Routing (QoSR) and FL on the networks to get better traffic management. Hence, after conducting this systematic survey, we have found an ample opportunity to the existing MPLS protocol for traffic engineering decisions by utilizing FL.

\section{The Proposed Fuzzy Control Methodology}

In this work, we have offered a soft component that classifies and arranging paths in preference order and then perform traffic splitting among them. The objective is to develop a system for highly loaded conditions i.e. when all communication links are congested and it is not possible to maintain QoS. The objective consists of categorising the paths in the preferential order and distributing the traffic over required number of LSP. FL is proved to be very effective in a lot of applications, such as intelligent control, decision making process etc. FL is based on a set of metrics which can be or not connected with each other. It calculates the best route by using minimum complex computation of the most important and appropriate parameters. The detail is in the next section.

\subsection{Architecture of Traffic Regulator: Fuzzy Logic Control System}

The Fuzzy logic Control System (FLCS) is sited at ingress node. FLCS is composed of two sub fuzzy control systems - Label Switched Path setup System (LsS) and Traffic Splitting System (TSS) as shown in the Figure 1.

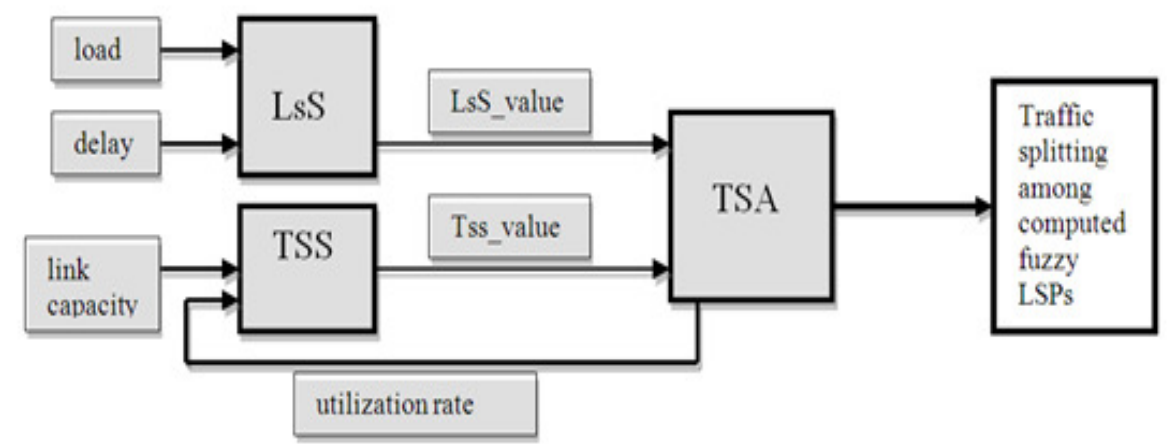

Figure 1.Computation of Fuzzy based LSPs to perform Traffic management

LsS selects LSPs according to two input fuzzy metrics (delay and load) and gives output fuzzy metric LsS_value. Load and Delay are suitable QoS factors on which selection of appropriate path depends. TSS performs the traffic splitting among the computed paths, according to the two 
input fuzzy metrics (utilization rate and link capacity) and gives output fuzzy metric TSS_value. An appropriate decision of traffic splitting is performed among the computed number of LSPs required by Traffic Splitting Algorithm (TSA). The computed fuzzy based LSPs for forwarding packets are obtained to avoid the situation of underutilization and over utilization of paths. None of the paths remains idle for longer time and proper utilization of resources takes place. Hence, it is better for congestion to be prevented rather than corrected. Implementation of FL using Mamdani Fuzzy Inference System [31] evaluates final decisions as LsS_value and TSS_value. The available number of rules in the Rule Base matrix LsS and TSS represents intermediate situations and provides the control mechanism with a highly dynamic action.

LsS rule base is a set of inputs (delay (D), load(L)) with their five linguistic values (Very Low (VL), Low (L), Medium (M), High (H), Very High (VH)) and output LsS_value with their seven linguistic values (Zero (Z), Tiny (T), Very Small (VS), Small (S), Big (B), Very Big (VB), High $(\mathrm{H})$ ) forms at most $5 * 5=25$ possible combinations. The matrix shown as Figure 2 is referred as Fuzzy Rule Base Matrix-LsS with columns representing D and rows representing L. Hence, optimized decisions are made using developed 25 rules (R1, R2, R3...R25), which are maximum possible cases for the selection of LSPs under different situations.

\begin{tabular}{|c|c|c|c|c|c|}
\hline load & VL & L & M & H & VH \\
\hline \multirow{2}{*}{ VL } & $\begin{array}{c}\text { Z } \\
\text { R1 }\end{array}$ & $\begin{array}{c}\text { T } \\
\text { R2 }\end{array}$ & $\begin{array}{c}\text { VS } \\
\text { R3 }\end{array}$ & $\begin{array}{c}\text { S } \\
\text { R4 }\end{array}$ & $\begin{array}{c}\text { B } \\
\text { R5 }\end{array}$ \\
\hline \multirow{2}{*}{ L } & T & VS & S & B & B \\
& R6 & R7 & R8 & R9 & R10 \\
\hline \multirow{2}{*}{ M } & T & VS & S & B & B \\
& R11 & R12 & R13 & R14 & R15 \\
\hline \multirow{2}{*}{ H } & VS & S & B & VB & VB \\
& R16 & R17 & R18 & R19 & R20 \\
\hline \multirow{2}{*}{ VH } & S & B & B & VB & H \\
& R21 & R22 & R23 & R24 & R25 \\
\hline
\end{tabular}

Figure 2. Fuzzy Rule Base Matrix- LsS with inputs (load, delay)

According to the QoS requirements of multimedia applications; indeed, the International Telecommunications Union standard (ITU- recommendation 14), we are defining boundaries of fuzzy sets of input parameters shown in Table 1.

Table 1. Linguistic values of (load, delay, utilization rate and link capacity) with their boundaries

\begin{tabular}{|c|c|c|c|c|c|c|c|}
\hline \multicolumn{8}{|c|}{ Linguistic values with their boundaries } \\
\hline \multirow{2}{*}{ Load } & VL & $\mathbf{L}$ & $\mathbf{M}$ & $\mathbf{H}$ & \multicolumn{3}{|c|}{$\mathrm{VH}$} \\
\hline & $\begin{array}{c}\text { Below } 0 \text { to } \\
250\end{array}$ & 0 to 500 & 250 to 750 & $\begin{array}{l}500 \text { to } \\
1000\end{array}$ & \multicolumn{3}{|c|}{750 to above than 1000} \\
\hline \multirow{2}{*}{ Delay } & VL & L & $\mathbf{M}$ & $\mathbf{H}$ & \multicolumn{3}{|c|}{ VH } \\
\hline & $\begin{array}{c}\text { Below } 0 \\
\text { to } .25\end{array}$ & 0 to 5.0 & 2.5 to 7.5 & 5.0 to 10 & \multicolumn{3}{|c|}{7.5 to above than 10} \\
\hline $\begin{array}{c}\text { Utilizatio } \\
\text { n rate }\end{array}$ & $\mathbf{Z}$ & $\mathbf{T}$ & VS & $\mathbf{S}$ & B & VB & $\mathbf{H}$ \\
\hline
\end{tabular}


International Journal in Foundations of Computer Science \& Technology (IJFCST) Vol.6, No.2, March 2016

\begin{tabular}{|c|c|c|c|c|c|c|c|}
\hline & $\begin{array}{c}\text { Below 0 } \\
\text { to .16 }\end{array}$ & 0 to .33 & .16 to .50 & .33 to .66 & $\begin{array}{c}.50 \\
\text { to } .83\end{array}$ & $\begin{array}{c}.66 \text { to } \\
1\end{array}$ & $\begin{array}{c}.83 \text { to } \\
\text { above than } \\
1\end{array}$ \\
\hline \multirow{2}{*}{$\begin{array}{c}\text { Link } \\
\text { capacity }\end{array}$} & VL & L & M & H & \multicolumn{2}{|c|}{ VH } \\
\cline { 2 - 8 } & $\begin{array}{c}\text { Below 0 to } \\
250\end{array}$ & 0 to 500 & 250 to 750 & $\begin{array}{c}500 \text { to } \\
1000\end{array}$ & 750 to above than 1000 \\
\hline
\end{tabular}

This FMM (LsS-Fuzzy Rule Base Matrix) provides large number of options to obtain best routing decisions. It provides efficient alternate paths in preference order that support Traffic Engineering and Path Optimization. The computed output response LsS_value identifies the paths as shown in Table 2.

It demonstrates the classification of LSPs and also defines the fuzzy set boundaries. Paths lying in region $\mathrm{Z}, \mathrm{T}, \mathrm{VS}, \mathrm{S}, \mathrm{B}, \mathrm{VB}, \mathrm{H}$ are best, very good, good, satisfactory, just acceptable, not acceptable and rejected respectively. Hence, in this way it arranges the paths in preferential order for the successful transmission of packets.

Table 2. Categorization of LSPs according to computed output value LsS_value

\begin{tabular}{|c|c|c|}
\hline $\begin{array}{c}\text { Paths } \\
\text { falling in } \\
\text { region }\end{array}$ & Category & Fuzzy set boundaries \\
\hline Z & Best & Less than 0 to .16 \\
\hline T & Very Good & 0 to .33 \\
\hline VS & Good & .16 to .50 \\
\hline S & Satisfactory & .33 to .66 \\
\hline B & Just & .50 to .83 \\
\hline VB & Not Acceptable & .66 to 1 \\
\hline H & Rejected & .83 to more than 1 \\
\hline
\end{tabular}

TSS rule base is a set of inputs (utilization rate (UR), link capacity (LC)), with their seven linguistic values of UR (Zero (Z), Tiny (T), Very Small (VS), Small (S), Big (B), Very Big (VB), High (H)) and five linguistic values of LC (Very Low (VL), Low (L) Medium (M), High (H), Very High $(\mathrm{VH})$ ) and output TSS_value with their five linguistic values (Very Low (VL), Low (L) Medium (M), High (H), Very High (VH)) forms at most 7*5 = 35 possible combinations. The matrix shown in Figure 3 is referred as Fuzzy Rule Base Matrix- TSS with columns representing UR and rows representing LC. Hence, optimized decisions are made using developed rules 35 (R1, R2, R3... R35). 
International Journal in Foundations of Computer Science \& Technology (IJFCST) Vol.6, No.2, March 2016

\begin{tabular}{|c|c|c|c|c|c|c|c|}
\hline & & & & & & & \\
\hline & Z & T & VS & S & B & VB & H \\
\hline link_capacity & & & & & & & \\
\hline VL & NC & NC & NC & NC & IO & IO & IW \\
& R1 & R2 & R3 & R4 & R5 & R6 & R7 \\
\hline L & NC & NC & NC & IO & IO & IW & IT \\
\hline M & R8 & R9 & R10 & R11 & R12 & R13 & R14 \\
\hline NC & Rl5 & R16 & R17 & R18 & R19 & R20 & R21 \\
\hline H & NC & IO & IW & IW & IT & IT & A \\
& R22 & R23 & R24 & R25 & R26 & R27 & R23 \\
\hline VH & IW & IW & IT & IT & A & A & A \\
& R29 & R30 & R31 & R32 & R33 & R34 & R35 \\
\hline
\end{tabular}

Figure 3. Fuzzy Rule Base Matrix-TSS with inputs (link capacity, utilization rate)

At different values of utilization rate and link capacity, the values of linguistic variables are obtained as defined in Table 1, which indicates the boundaries of input fuzzy sets of TSS. This FMM (TSS-Fuzzy Rule Base Matrix) gives the information of the requirement of number of LSPs. Table 3 demonstrates the number of LSPs needed for the operation according to traffic scenario. If TSS_value lies in the region of NC, it is defined as no change or no need of additional LSP for congestion free transmission. Requirement of LSPs is obtained from TSS_value lies as defined in Table 3, to check that in which region (IO, IW, IT, A) values are lying.

Table 3. Traffic Splitting among LSPs according to output value TSS_value

\begin{tabular}{|c|c|c|}
\hline $\begin{array}{c}\text { Splitting } \\
\text { requirement } \\
\text { falling in } \\
\text { Region }\end{array}$ & $\begin{array}{c}\text { Requirement of } \\
\text { LSPs }\end{array}$ & Fuzzy set boundaries \\
\hline NC & $\begin{array}{c}\text { No Change or no } \\
\text { need of additional } \\
\text { LSP }\end{array}$ & Below than 0 to .25 \\
\hline IO & Increase by one & 0 to .50 \\
\hline IW & Increase by two & .250 to .83 \\
\hline IT & Increase by three & .50 to 1 \\
\hline A & All available LSPs & .83 to more than 1 \\
\hline
\end{tabular}

The requirement of number of LSPs depends upon the current dynamic scenario of the network in terms of highly loaded, loaded, equilibrium, lightly loaded, and least loaded. In highly loaded network - more number of paths is required and in lightly loaded- less number of paths is required. Hence, by applying Traffic Engineering (TE)/load balancing, objective of congestion free network is achieved.

For the simplicity of operation, Triangular Membership Function (TMF) is applied to obtain the membership function of metrics as:-

$$
\text { triangle }(x ; a, b, c)=\max (\min (x-a / b-a, c-x / c-b), 0)
$$


It is defined by three parameters, where $b$ indicates the point on which, the membership function value is 1 , a and $\mathrm{c}$ indicate the left and right limits of the definition domain of the membership function. On applying TMF as shown in formula (1), we obtain membership functions of input and output parameters as follows:-

$\mu_{\text {load }}=\left\{\mu_{\mathrm{VL}}, \mu_{\mathrm{L}}, \mu_{\mathrm{M}}, \mu_{\mathrm{H}}, \mu_{\mathrm{VH}}\right\}$

$\mu_{\text {delay }}=\left\{\mu_{\mathrm{VL}}, \mu_{\mathrm{L}}, \mu_{\mathrm{M}}, \mu_{\mathrm{H}}, \mu_{\mathrm{VH}}\right\}$

$\mu_{\text {LsS_value }}=\left\{\mu_{\mathrm{Z}}, \mu_{\mathrm{T}}, \mu_{\mathrm{VS}}, \mu_{\mathrm{S}}, \mu_{\mathrm{B}}, \mu_{\mathrm{VB}}, \mu_{\mathrm{H}}\right\}$,

$\mu_{\text {utilization_orate }}=\left\{\mu_{\mathrm{Z}}, \mu_{\mathrm{T}}, \mu_{\mathrm{vS}}, \mu_{\mathrm{S}}, \mu_{\mathrm{B},} \mu_{\mathrm{VB},}, \mu_{\mathrm{H}}\right\}$,

$\mu_{\text {link_capacity }}=\left\{\mu_{\mathrm{VL}}, \mu_{\mathrm{L}}, \mu_{\mathrm{M}}, \mu_{\mathrm{H}}, \mu_{\mathrm{VH}}\right\}$,

$\mu_{\mathrm{TSS} \_ \text {value }}=\left\{\mu_{\mathrm{NC}}, \mu_{\mathrm{IO}}, \mu_{\mathrm{IW}}, \mu_{\mathrm{IT}}, \mu_{\mathrm{A}}\right\}$.

Decisions are based on the testing of rules of the rule bases and recognition of fired rules (activated rules). Minimum (MIN) operation is applied to find out the minimum or least value of parameters. The output responses LsS_value and TSS_value are obtained by applying logical product (AND) on fired rules. These fired rules combined to make an optimal decision. Composition combines the effects of all applicable rules and gives the best-weighted influence of fired rules as shown in Table 4. Output linguistic terms are obtained as the combination of different rules. The execution of if then rules of fuzzy controllers is explained in Annexure I.

Table 4. Composition of rules of fuzzy controllers LsS and TSS

\begin{tabular}{|l|l|}
\hline & Output linguistic variables \\
\hline & $\mathrm{Z}=\mathrm{R} 1$ \\
$\mathrm{~T}=\mathrm{R} 2+\mathrm{R} 6+\mathrm{R} 11$ \\
$\mathrm{~S}=\mathrm{R} 3+\mathrm{R} 7+\mathrm{R} 12+\mathrm{R} 16$ \\
$\mathrm{LsS}$ & $\mathrm{VS}=\mathrm{R} 4+\mathrm{R} 8+\mathrm{R} 13+\mathrm{R} 17+\mathrm{R} 21$ \\
$\mathrm{~B}=\mathrm{R} 5+\mathrm{R} 9+\mathrm{R} 10+\mathrm{R} 14+\mathrm{R} 15+\mathrm{R} 18+\mathrm{R} 22+\mathrm{R} 23$ \\
$\mathrm{VB}=\mathrm{R} 19+\mathrm{R} 20+\mathrm{R} 24$ \\
$\mathrm{H}=\mathrm{R} 25$
\end{tabular}

Decision function is computed using as Center Average defuzzifier or discrete centroid method as follows:-

$$
\mu_{C A}=\sum \eta_{i=1} \mu_{x i} * X_{c i} / \sum_{i=1} \mu_{y i}
$$

This is an approximation of Center of Area (COA) defuzzifier method [33, 34]. $\mathrm{x}_{\mathrm{ci}}$ denotes center points of the output linguistic terms $(x i), \mu_{\mathrm{CA}}$ is membership function of output linguistic term and $\mu_{x i}$ is membership function value of input linguistic term. On applying formula (2) on the linguistic terms and linguistic centers of control systems, LsS_value and TSS_value are obtained as shown in Table 5 . 


\begin{tabular}{|c|c|c|c|}
\hline & $\begin{array}{l}\text { Linguis } \\
\text { tic term }\end{array}$ & $\begin{array}{l}\text { Linguis } \\
\text { tic } \\
\text { centre }\end{array}$ & \\
\hline LsS & \begin{tabular}{|l}
$\mathrm{Z}$ \\
$\mathrm{T}$ \\
$\mathrm{VS}$ \\
$\mathrm{S}$ \\
$\mathrm{B}$ \\
$\mathrm{VB}$ \\
$\mathrm{H}$ \\
\end{tabular} & \begin{tabular}{|l}
$\mathrm{Z}_{\mathrm{C}}$ \\
$\mathrm{T}_{\mathrm{C}}$ \\
$\mathrm{VS}_{\mathrm{C}}$ \\
$\mathrm{S}_{\mathrm{C}}$ \\
$\mathrm{Bc}$ \\
$\mathrm{YBc}$ \\
$\mathrm{VH}_{\mathrm{C}}$ \\
\end{tabular} & $\begin{array}{l}\text { LsS_value }=\left(\mathbf{Z}^{*} \mathbf{Z}_{\mathrm{C}}+\mathrm{T}^{*} \mathrm{~T}_{\mathrm{C}}+\mathrm{VS}^{*} \mathrm{VS}_{\mathrm{C}}+\mathrm{S}^{*} \mathrm{~S}_{\mathrm{C}}+\mathrm{B}^{*} \mathrm{~B}_{\mathrm{C}}+\mathrm{VB}^{*} \mathrm{VB}_{\mathrm{C}}\right. \\
\left.+\mathrm{H}^{*} \mathrm{H}_{\mathrm{C}}\right) /(\mathrm{Z}+\mathrm{T}+\mathrm{VS}+\mathrm{S}+\mathrm{B}+\mathrm{VB}+\mathrm{H})\end{array}$ \\
\hline TSS & $\begin{array}{l}\text { NC } \\
\text { IO } \\
\text { IW } \\
\text { IT } \\
\text { A }\end{array}$ & $\begin{array}{l}\mathrm{NC}_{\mathrm{C}} \\
\mathrm{IO}_{\mathrm{C}} \\
\mathrm{IW}_{\mathrm{C}} \\
\mathrm{IT}_{\mathrm{C}} \\
\mathrm{A}_{\mathrm{C}}\end{array}$ & $\begin{array}{l}\text { TSS_value }=\left(\mathbf{N C} * \mathrm{NC}_{\mathrm{C}}+\mathrm{IO}^{*} \mathrm{IO}_{\mathrm{C}}+\mathrm{IW}^{*} \mathrm{IW}_{\mathrm{C}}+\mathrm{IT}^{*} \mathrm{IT}_{\mathrm{C}}+\mathrm{A}^{*} \mathrm{~A}_{\mathrm{C}}\right) / \\
(\mathrm{NC}+\mathrm{IO}+\mathrm{IW}+\mathrm{IT}+\mathrm{A})\end{array}$ \\
\hline
\end{tabular}

\subsection{Decision maker: Fuzzy Logic Control System Algorithm (FLCS)}

FLCS algorithm is a proposed algorithm which implements and evaluates results of above mentioned system. It is composed of two parts; firstly determination and selection of multiple paths using LsS and secondly allocating network traffic among the required number of paths using TSS.

\subsubsection{Determination and Selection of multiple paths using LsS}

It uses two metrics L, D for the selection of LSPs. $L(i, j)$, load specifying number of packets to be sent on link $(\mathrm{i}, \mathrm{j})$ and $D(i, j)$, delay specifying delay of link $(\mathrm{i}, \mathrm{j})$ during transmission. $D(i, j)$ is computed as follows:-

$$
D(i, j)=L(i, j) / L C(i, j) .
$$

LsS fuzzy inference system gives $L s S \_$value $(i, j)$, which identifies links for the selection of LSPs. Link having minimum LsS_value is selected for the operation.

\subsubsection{Allocating network traffic among the required number of paths using TSS.}

It uses two metrics LC and UR for finding the requirement of LSPs for successfully congestion free transmission. It is considered that link capacity of each link will remain same throughout the execution, but load, delay, utilization rate get changed. $L C(i, j)$, link_capacity giving bandwidth value of link $(\mathrm{i}, \mathrm{j}) . U R(i, j)$, utilization_rate specifying utilization rate of link $(\mathrm{i}, \mathrm{j})$ computed as

$$
\operatorname{UR}(\mathrm{i}, \mathrm{j})=\operatorname{Freq}(\mathrm{i}, \mathrm{j}) / \mathrm{NL}(\mathrm{i}) \text {. }
$$

Where Freq $(i, j)$ is a frequency of link $(\mathrm{i}, \mathrm{j})$ and $N L(i)$ gives a number of outgoing links from node i. TSS fuzzy inference system gives TSS_value $(i, j)$ of each link used to compute number of paths required (Nlsps) for operation. The execution steps of FLCS algorithm shown below. 


\section{$\operatorname{FLCS}(L, D, U R, L C)$}

Begin

1. LsS_value $(i, j)=\operatorname{LsS}(D, L) \quad / *$ Call LsS computes LsS_value to identify links for the selection of LSPs on processing inputs $D$ and $L * /$

2. TSS_value $(i, j)=T S S(U R, L C)$

Nlsps $=$ TSS_value $(i, j) \quad / *$ Call TSS computes TSS_value to obtain the number of LSPS required for operation on processing input UR and $L C *$ /

3. while $(L(i, j) !=0) \quad / *$ Transmission will be continue till load at node $i=$ ingress is non zero*/

3.1 for $k=1$ to Nlsps $\quad / *$ Traffic splitting takes place among the Nlsps */

$\operatorname{Slsp}[k]=\min (\operatorname{LsS}(i, j)) \quad / *$ Generation of optimal LSPS (links having minimum

$* /$

LsS_value) is obtained in Slsp

End

$$
3.2 \text { Freq }(i, j)=\text { Freq }(i, j)+1 \quad / * \text { Respective frequency of LSP is updated } *
$$

Step 1:-LsS computes the respective LsS_value of each link (i,j) of FLCS.

Step 2:-TSS computes the respective TSS_value of each link $(\mathrm{i}, \mathrm{j})$ of FLCS, which gives the requirement of traffic splitting in network.

Step 3:-Transmission will continue till load at ingress is non zero. Traffic splitting takes place among the optimal Nlsps (LSP having minimum LsS_value). Respective frequency of each link $(\mathrm{i}, \mathrm{j})$ is incremented according to the number of times a particular link (i,j) is used. Slsp gives the optimal LSPs for operation.

\section{An Illustrative Example to Realize Algorithm}

For the realization of FLCS, the configuration of the network of 8 nodes shown in Figure 4 is assumed. The ingress of network is node 1 and egress is node 8 . In the network each link $(\mathrm{i}, \mathrm{j})$ contains information as a set of 4 parameters (L, D, UR, LC). The algorithm presented in section 3.2 is executed for the assumed values of parameters shown as coordinates of links in Figure 4. Membership functions are obtained for all the set of values of links of assumed network using formula $1 \& 2$. In section 4.1, algorithm is implemented and results are obtained. It section 4.2, the demonstration of generation of LSPs using proposed approach is described.

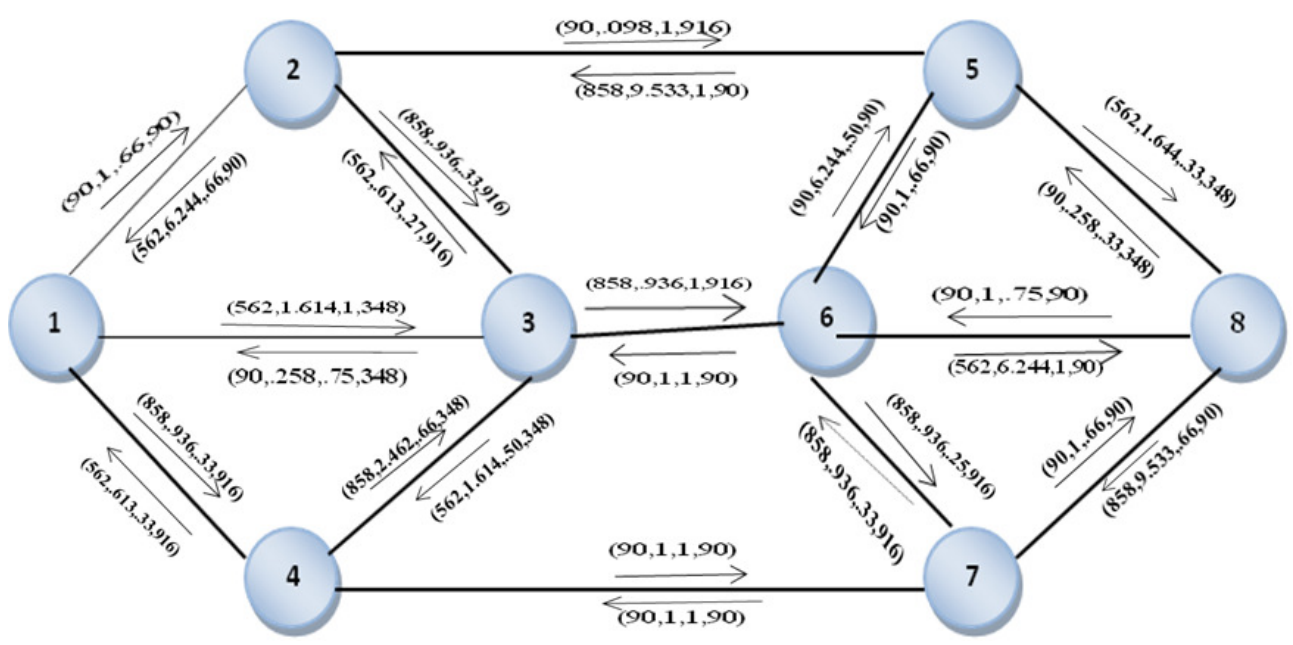

Figure 4. Input values of L, D, UR, and LC 


\subsection{Realization of an Algorithm}

At a particular instance membership function value of input parameters $L, D, U R, L C$ are assumed as $\mathrm{L}=858 \mathrm{Mb}, \mathrm{D}=.936 \mathrm{~s}, \mathrm{UR}=.33 \mathrm{Mb} / \mathrm{s}, \mathrm{LC}=916 \mathrm{Mb} / \mathrm{s}$ as shown in Annexure II, using Step 1 and Step 2, values of output parameters LsS_value and TSS_value at these respective input parameters are evaluated. The final decision as LsS_value obtained indicates that the particular link is $86 \%$ in S and 14\% in VS as displayed in Figure 7 of Annexure II.

The final decision as TSS_value obtained indicates that according to the network status $32 \%$ increase of LSPs by two and 68\% increase of LSPs by three are required VS as displayed in Figure 8 of Annexure II. It concludes that the selected link needs $68 \%$ splitting of traffic by increasing LSPs by three. Throughout the simulation process, the updated information of all links is maintained according to which final decisions takes place. For the better understanding of approach, we have executed FLCS with different parametric values. The predictions of each link are computed for the selection of appropriate links for operation. The observations at three different instants are obtained, which depicts that paths are best, good, very good, satisfactory, just acceptable, not acceptable and rejected according to the dynamic network. Hence, our algorithm has all the essential requirements into its account. The results are obtained from fuzzy controller (LsS, TSS) as matrix LsS_value and TSS_value.

\subsection{Generation of LSPS in Assumed Scenario}

The generation of LSPs takes place by the execution of FLCS algorithm as studied in above section.

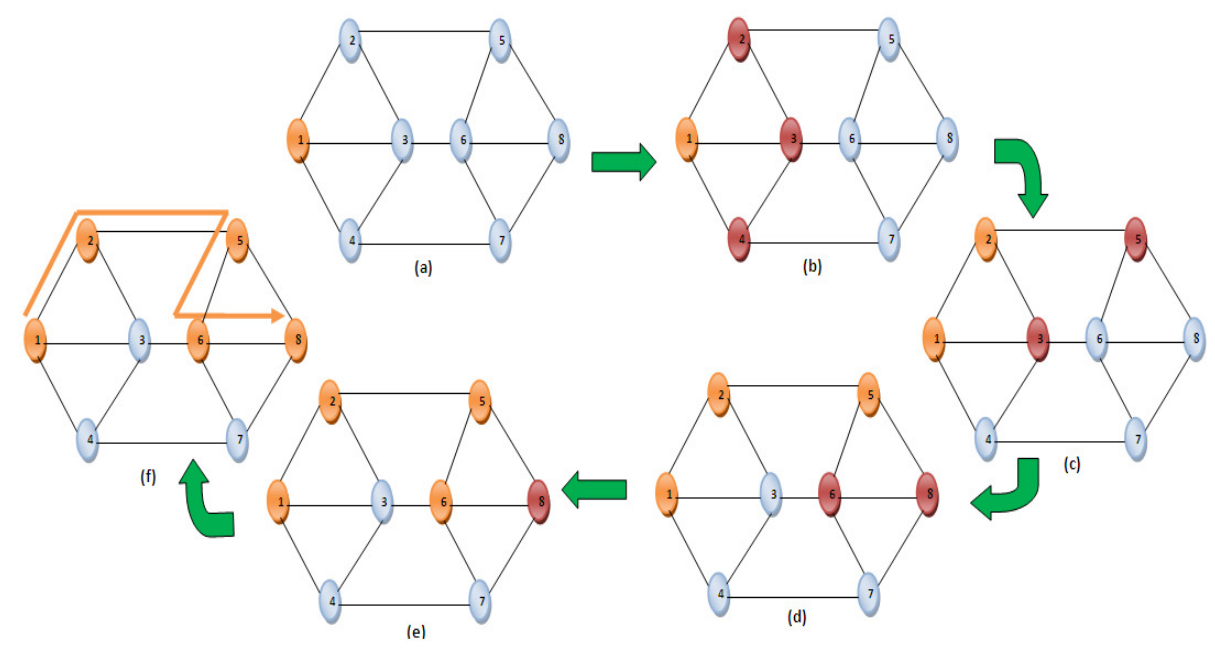

Figure 5. Generation of LSPs

In Figure 5(a), node 1 is colored orange it indicates that it is a source. Node 1 is having neighbors as node 2, node3 and node 4. So, these are colored red as shown in Figure 5(b) and the LsS_value of neighbors are compared. In Figure 5(c) node 2 is colored orange which indicates that it is selected as it is having least LsS_value. Node 2 is having neighbors as node 3 and node 5 as shown in Figure 5(c) and the LsS_value of neighbors are compared. Node 5 is colored orange which indicates that it is selected as it is having least LsS_value shown in Figure 5 (d). Node 5 is having neighbors as node 6 and node 8 as shown in Fig.5 (d) and the LsS_value of neighbors are compared. Node 8 is colored orange which indicates that it is selected as it is having least LsS_value shown in Figure 5 (e), which is final destination also. Hence, in this manner LSP is 
generated from node 1 to node 8 as shown in Figure 5 (f). Similarly other LSPs are generated; list of paths in preference order at different values of parameters is generated as shown in Table 6.

Table 6. Generation of LSPs in the network of 8 nodes

\begin{tabular}{|l|l|l|l|l|}
\hline \multirow{2}{*}{ Node } & \multicolumn{5}{|c|}{ Generated LSPs in preference order } \\
& \multicolumn{4}{|c|}{ Ingress = 1 and Egress $=8$} \\
\hline 1 & $1->2->5->6->8$ & $1->3->4->7->8$ & $1->4->7->8$ & \\
\hline 2 & $2->5->6->8$ & $2->3->4->7->8$ & $2->3->6->8$ & $2->3->6->7->8$ \\
\hline 3 & $3->4->7->8$ & $3->6->8$ & $3->6->7->8$ & \\
\hline 4 & $4->7->8$ & & & \\
\hline 5 & $5->6->8$ & $5->8$ & $5->6->7->8$ & \\
\hline 6 & $6->8$ & $6->7->8$ & & \\
\hline 7 & $7->8$ & & & \\
\hline
\end{tabular}

Values of parameters change the decision of selection of path. As, we have to use the optimal paths for the operation. Generated paths are optimal in terms of number of hops, in order to minimize the network cost. It has been observed that, when value of these parameters increases beyond the limit then path becomes unsuitable for future use. Dynamic status of network is studied and according to that suitable paths are selected. The proposed algorithm is justifying the requirement specifications of MPLS algorithm as described in [34] and discussed in previous study too. The proposed algorithm is an online routing algorithm; computational expenses are low and work on the QoS metrics, which is the most desirable feature.

\subsection{Attained Observations of Three Different Scenarios}

For validating incremental progress related to solving the problem of traffic splitting, a comparison between classical approach (OSPF) [39] and proposed work (FLCS) was conducted by multiple experiments using NS-3[35]. The scenario composed of MPLS-TE together with constraint based routing (CBR). FLCS performs the dynamic route selection. Our work demonstrates the improvements by using FLCS for LSP Selection. The topology used to evaluate the proposed work behaviour consists of three different link capacities and propagation delay, a) $10 \mathrm{Mbps}, 3 \mathrm{~ms}$, b) $100 \mathrm{Mbps}, 2 \mathrm{~ms}$, c) $1000 \mathrm{Mbps}, 1 \mathrm{~ms}$. The decisions will be taken by FLCS, which works in ingress node. The incoming request follows an exponential distribution and the requested bandwidth is uniformly distributed between ranges [0 10] (Mbps), [10 100] (Mbps), [100 1000] (Mbps) to model Voice, Data and Video Traffic respectively [36- 38]. Holding time is randomly distributed with a mean of $300 \mathrm{sec}(\mathrm{ON}=0.325 \mathrm{OFF}=.64)$. IPV4 network stack has been used and packets are generated using a hypothetical ON/OFF model using the Drop tail queue. Different traffic types are used in simulations like the FTP over the TCP to simulate data traffic and the constant bit rate (CBR) to simulate voice and video over the UDP. The packet size of the User Datagram Protocol (UDP) is 512 byte and 1024 byte for the Transport Control Protocol (TCP). Finally, the existing topology and an available resources present in the database of ingress are perfectly updated. The statistical comparison (MAXIMUM (MAX), MINIMUM (MIN), and STANDARD DEVIATION (STDEV)) of FLCS and OSPF is depicted in Table 7, which reflects that standard deviation for different traffic is high in the case of OSPF. 
International Journal in Foundations of Computer Science \& Technology (IJFCST) Vol.6, No.2, March 2016

Table 7. Statistical study of the results obtained OSPF (red) and FLCS (blue)

\begin{tabular}{|c|c|c|c|c|c|c|c|c|}
\hline \multicolumn{3}{|c|}{ Video Traffic } & \multicolumn{3}{|c|}{ Data Traffic } & \multicolumn{3}{|c|}{ Voice Traffic } \\
\hline \multicolumn{9}{|c|}{ Mean Loss Rate } \\
\hline & OSPF & FLCS & & OSPF & FLCS & & OSPF & FLCS \\
\hline MAX & 33.6 & 29.4 & MAX & 0.16 & 8.604 & MAX & 19.1 & 14.9 \\
\hline MIN & 0 & 0 & MIN & 0.0011 & 0.001 & MIN & 0 & 0 \\
\hline STDEV & 9.66351 & 8.98499 & STDEV & 2.95828 & 3.467 & STDEV & 4.50338 & 5.41680 \\
\hline \multicolumn{9}{|c|}{ Mean Delay } \\
\hline & OSPF & FLCS & & OSPF & FLCS & & OSPF & FLCS \\
\hline MAX & 0.96 & 0.04 & MAX & 0 & 0.126 & MAX & 0.25 & 0.21 \\
\hline MIN & 0 & 0 & MIN & 0.0001 & 0 & MIN & 0 & 0 \\
\hline STDEV & 0.0193 & 0.01888 & STDEV & 0.0477 & 0.399 & STDEV & 0.0707 & 0.8286 \\
\hline
\end{tabular}

In the Figure $6(a, b)$ the mean delay and the mean loss rate of video traffic is displayed, which is obtained with varying UDP traffic for 300 seconds. In the Figure $6(\mathrm{c}, \mathrm{d})$ the mean delay and mean loss rate of data traffic is displayed, which is obtained with varying UDP traffic for 300 seconds. In the Figure $6(\mathrm{e}, \mathrm{f})$ the mean delay and the mean loss rate of voice traffic is displayed, which is obtained with varying UDP traffic for 300 seconds. It exhibits improvements in the terms of mean delay $(42.0 \%)$ and mean loss rate $(2.4 \%)$ for Video Traffic, mean delay $(5.4 \%)$ and mean loss rate (3.4\%) for Data Traffic and mean delay (44.9\%) and mean loss rate(4.1\%) for Voice Traffic. Moreover, $42 \%, 15.4 \%$ and $44.4 \%$ of Video packets, Data packets and Voice packets respectively are dropped due to buffer overflow at the congested node when using OSPF. FLCS on the other hand, as seen from Figure 6 by avoiding this congestion as mentioned earlier, maintains the traffic delivery performance. By distributing the traffic over suitable preferable paths and maintaining low queue lengths, all the packets can be successfully delivered.

The result is an increase in the queuing delay at a particular congested node, consequently leading congestion, and large waiting time at a few hops where the performance is poor. For few seconds in the above scenarios, OSPF outperforms due to low queuing delay. Even though FLCS utilizes multiple paths and it leads to less delay for the said congested path. The obtained results of simulation experiments are displayed in Table 7 and Figure 6 that give a better understanding of research work. These findings predict that the fuzzy traffic monitor proved as more efficient solution and constitute a good alternative to some conventional methods of traffic engineering based on complex optimization.

\section{Conclusions}

Our main premise of this study is based on the fuzzy traffic regulation which works within the realms of MPLS. The FLCS is sited at ingress node. The proposed fuzzy based approach is highly suitable for congestion control over MPLS networks. It applies QoS \& TE constraints. It is designed for better video delivery, since it provides better throughput, average delay. We have also empirically demonstrated the advantages of FLCS over OSPF in NS-3.

In current simulation, the network was using 2 edge routers and 6 switch routers. We have displayed the obtained results and predictions in above sections. It is applicable for any kind of traffic, where traffic volume is small and traffic type is normal. However, it is based on traffic scenarios, which have been implemented in this research paper. It exhibits improvements in the terms of mean delay $(42.0 \%)$ and mean loss rate $(2.4 \%)$ for Video Traffic, mean delay $(5.4 \%)$ and mean loss rate (3.4\%) for Data Traffic and mean delay (44.9\%) and mean loss rate $(4.1 \%)$ for 
Voice Traffic. Moreover, 42\%, 15.4\% and $44.4 \%$ of Video packets, Data packets and Voice packets respectively are dropped due to buffer overflow at the congested node when using OSPF. This work gives an opportunity to analyze the complex scenario in network traffic management, which would help future implementation in this context. The proposed approach has very simplified computation as at most 4 rules are fired at an instance. Hence, 4-5 additions and multiplications are required. Only edge routers are required to be fuzzy inference engine capable, which needs few kilobytes of memory.

The proposed approach has been presented by an example and it has been concluded that when the value of $\mathrm{L}, \mathrm{D}$ changes then, the selection of path also changes. The varying values of UR, LC determines the traffic splitting among required number of paths. The results presented in this paper suggest that this fuzzy logic system can accommodate to the traffic conditions and decide the necessary action accordingly.

\section{FUTURE DiRECTIONS}

In Future, work can be carried out to validate the approach in more simulated dynamic scenarios. The interest of such a work has a big impact for the applications related to the networks, in particular those dedicated to the mission critical applications. We plan to improve/extend the work by investigating the practicality of combining multiple learning methods to perform traffic regulation. In conclusion, multipath routing and provision continue to underlie the current internet and will shape its future and further innovation.

\section{ACKNOWLEDGEMENTS}

We are highly thankful to the reviewers for their valuable suggestions and comments that significantly helped in improving the quality of research paper. 
International Journal in Foundations of Computer Science \& Technology (IJFCST) Vol.6, No.2, March 2016

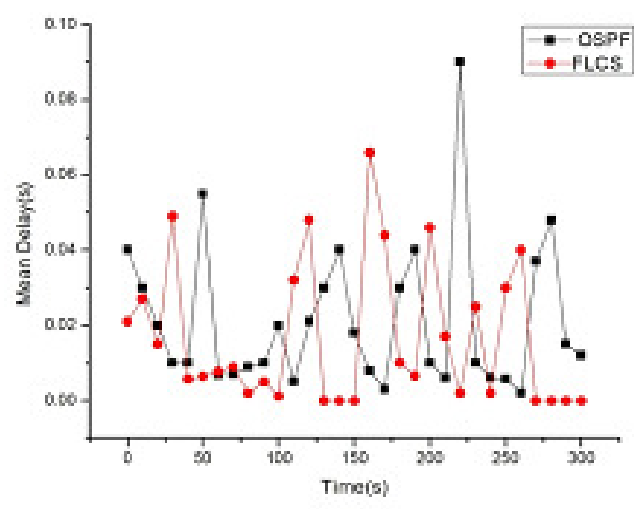

(a)

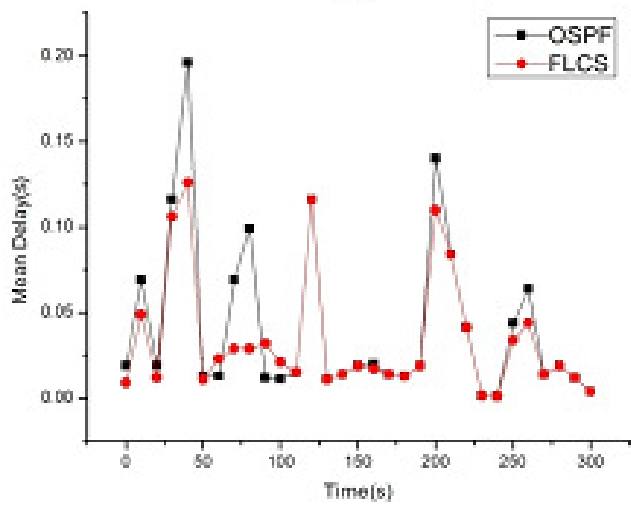

(c)

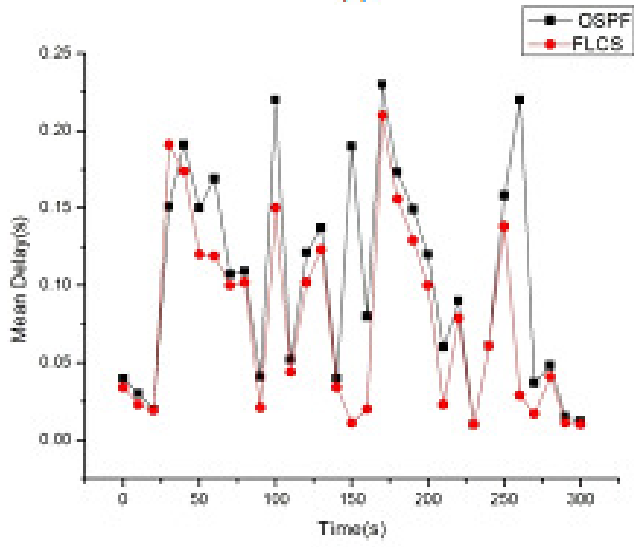

(e)

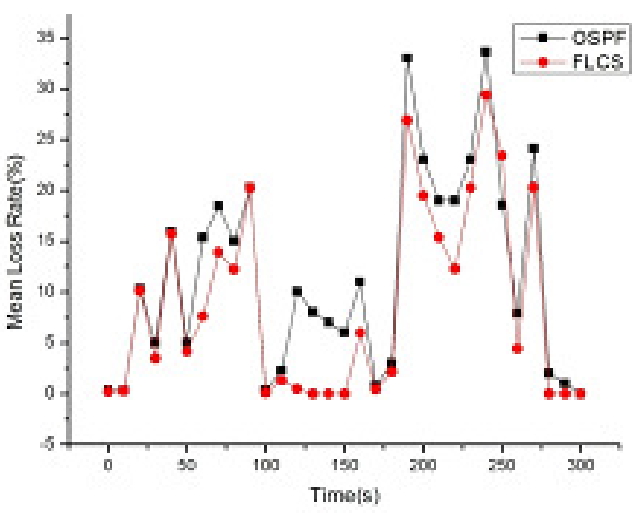

(b)

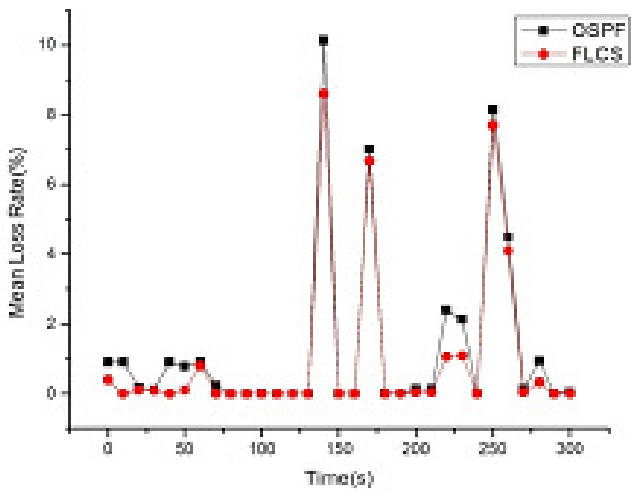

(d)

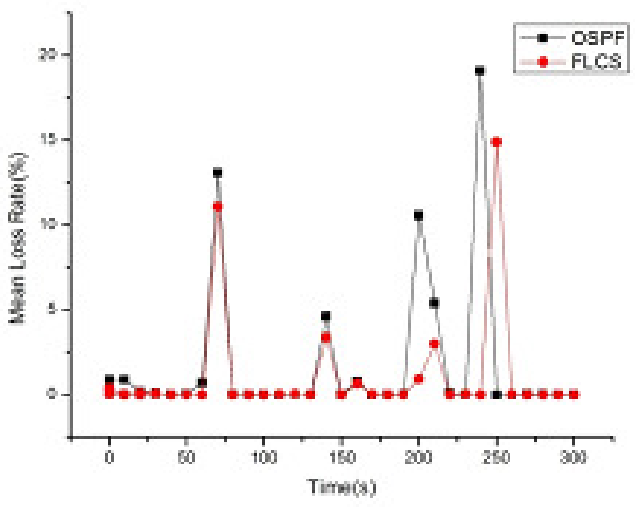

(f)

Figure 6. Variation of Mean delay (s) and Mean Loss Rate (\%) with respect to time (secs) for (a) (b) Video Traffic (c) (d) Data Traffic (e) (f) Voice Traffic

\section{REFERENCES}

[1] E.Rosen, A.Viswanathan and R.Callon, (2001), Multi-protocol Label Switching Architecture, RFC 3031.

[2] C.Siva Ram, Murthy Mohan Guruswami, 2002, WDM Optical Networks Concepts, Design and Algorithms, Prentice Hall of India.

[3] Jeremy Lawrence, (2001), Designing Multiprotocol Label Switching Networks, IEEE Communications Magazine, 134-142. 
[4] D.Awduche, (1999), MPLS and Traffic Engineering in IP Networks, IEEE Communications Magazine, 37(12), 42-47.

[5] Genge, B., \& Siaterlis, C. (2013). Analysis of the effects of distributed denial-of-service attacks on MPLS networks. International Journal of Critical Infrastructure Protection, 6(2), 87-95.

[6] G.Swallow, MPLS Advantages for Traffic Engineering, IEEE Communications Magazine, 37(12), (1999), 54-57.

[7] Z.Wang and J. Crowcraft, (1996), Quality-of-Service routing for supplying multimedia applications, IEEE journal on Selected Areas in Communication, 14(1996), 1228-1234.

[8] Murali S.Kodialam, T. V. Lakshman, (2003), Dynamic Routing of Restorable Bandwidth-Guaranteed Tunnels Using Aggregated network resource Usage Information, IEEE/ACM Transactions on Networking, 11, 399-410.

[9] Q.Zheng, G.Mohan, (2006), LSP protection for delay-differentiated dynamic traffic in IP-over-WDM networks with port constraints, Computer Communications, 29, 1402-1412.

[10] Scott Fowler, Sherali Zeadally and Abdelhamid Mellouk, (2010), End-to End QoS Engineering in next Generation heterogeneous Networks, Wiley Online Library, 309-345.

[11] Wu-Hsiao Hsu, Yuh- Pyng Shieh and Jenhui Chen, (2010), Multiple path selection algorithm for DiffServ-Aware MPLS traffic engineering, Elsevier Science Journal Computer Communications, 33, 1557-1565.

[12] Chaun Hau Lau, (2006), Path selection with preemption and re-routing control for multiprotocol label switching networks, Computer Communications 29, 3719-3732.

[13] Mohammad Hossien Yaghmaee, Mohammad bagher Menhaj, (2001), A novel FLC-based approach for ATM traffic control, Computer Networks, 36, 643-658.

[14] Mohammad Hossien Yaghmaee,Hoda Taheri, Peyman Neakatollahi, (2012),An energy-aware distributed clustering protocol in wireless sensor networks using fuzzy logic, Ad-Hoc Networks $10,1469-1481$.

[15] C. Chrysostomou, A. Pitsillides, L. Rossides, A. Sekercioglu, (2003), Fuzzy Logic Controlled RED: Congestion control in TCP/IP differentiated services networks, Soft Computing $8,79-92$.

[16] Edgard Jamhour, Manoel C.Penna,Ricardo Nabhen, (2009), Modeling a multi-queue network node with a fuzzy predictor, Fuzzy Sets and Systems, 160,1902-1928.

[17] Mohammad Hossien Yaghmaee, Mohammad bagher Menhaj, Design and performance evaluation of a fuzzy based traffic conditioner for differentiated services, Computer Networks 47(2005), 847-869.

[18] Junaid A. Khan, Hussein Alnuweiri, (2003), A Traffic Engineered Routing Algorithm Based on Fuzzy Logic, IEEE Pacific Rim Conference on Communications, Computers and Signal Processing, 1,454-457.

[19] Junaid A. Khan, Hussein Alnuweiri, (2004), A Fuzzy Constraint-Based Routing Algorithm for Traffic Engineering, IEEE Communications Society1366-1372.

[20] E.Aboelela, C.Douligeris, (1998), Fuzzy Multiobjective Routing Model In B-1SDN, Journal of Computer Communication, 21(17), 1572-1585.

[21] E.Aboelela, C.Douligeris, July(1997), Routing in Multimetric Networks Using A Fuzzy Link Cost, The Second IEEE Symposium on Computers and Communications ,397-401.

[22] M.H.Yaghmaee, M.Bahekmat, G. Khojasteh Toussi, (2008), A Novel Fuzzy Logic Base Scheduling Mechanism for Service Differentiation in IP Networks, Journal of Iranian Association of Electrical and Electronics Engineers $52,54-64$.

[23] N.M.Din and N.Fisal, (2008), Fuzzy Logic Bandwidth Prediction and Policing in A DiffServ-Aware Network, Journal of Computers 3(5), 18-20.

[24] Joaguim Celestino Junior, (2004), FuDyLBA: A Traffic Engineering Load Balance Scheme for MPLS Networks Based on Fuzzy Logic, Springer LNCS 3124, 622-627.

[25] G.Ran, H.Zhang, S.Gong, (2010), Improving on LEACH protocol of Wireless Sensor Networks using Fuzzy Logic, Journal of Information and Computational Science, 767-775.

[26] B.Chen, Y.Yang, B.Lee, T.Lee, (2003) ,Fuzzy adaptive predictive flow control of ATM network traffic, IEEE Transactions on Fuzzy Systems 11 (4),568-581.

[27] S.Ghosh, Q.Razouqi, H.J.Schumacher, A. Celmins, A survey of recent advances in fuzzy logic in telecommunications networks and new challenges, IEEE Trans. Fuzzy Syst. 6 (3) (1998), 443-447.

[28] Nahid Ebrahimi Majd, Mohammad Hossien Yaghmaee, (2006), A Fuzzy Algorithm for QoS-Based Routing in MPLS Network, IEEE, Asia-Pacific Conference, APCC '06.

[29] N.M.Din and H.Z.Abidin, S.F.A.Rahman and N.Fisal, (2005), A Fuzzy LSP Regulator for Preemption Control in A DiffServ-Aware MPLS Internet, IEEE 7th Malaysia International Conference , 415-420. 
[30] Sergio Gramajo, Luis Martinez, (2012), A linguistic decision support model for QoS priorities in networking, Knowledge-Based Systems 32, 65-75.

[31] J.S.R.Jang, C.T.Sun, E.Mizutani, Neuro-Fuzzy and Soft Computing, Pearson Education, 2010.

[32] D.O.Awduche, J.Malcom, J.Agogbua, M. O’D` ell, and J. McManus, Sept.1999,” Requirements for traffic engineering over MPLS," RFC 2702.

[33] E.V.Broekhoven and B.D.Baets, 2006, "Fast and accurate center of gravity defuzzification of fuzzy system outputs defined on trapezoidal fuzzypartitions,"Fuzzy Sets Syst., vol.157, no. 7, pp. 904-918.

[34] W.V.Leekwijck and E.E.Kerre, 1999, "Defuzzification: Criteria and classification, "Fuzzy Sets Syst., vol. 108, no. 2, pp. 159-178.

[35] Carneiro, G., Fontes, H., \& Ricardo, M. (2011). Fast prototyping of network protocols through ns-3 simulation model reuse. Simulation Modeling Practice and Theory, 19(9), 2063-2075.

[36] Sánchez-López, S., Masip-Bruin, X., Solé-Pareta, J., \& Domingo-Pascual, J. (2002). Providing QoS in MPLS-ATM integrated environment. In From QoS Provisioning to QoS Charging (pp. 215-224). Springer Berlin Heidelberg.

[37] Adas, A.(1997). Traffic models in broadband networks. Communications Magazine, IEEE, 35(7), 8289.

[38] Park, K., \& Willinger, W.(2000). Self-similar network traffic: An overview. Self-similar network traffic and performance evaluation, 1-38.

[39] Henderson, T.R., Roy, S., Floyd, S., \& Riley, G. F. (2006, October). ns-3 project goals. In Proceeding from the 2006 workshop on ns-2: the IP network simulator (p. 13). ACM.

\section{Authors}

Ms. Anju Bhandari is pursuing doctoral program $(\mathrm{PhD})$ at Computer Science and Engineering Department of Thapar University, Patiala, India. Her qualifications include B.Tech (CSE), M.Tech (CSE). She is member of ISTE. She has 9 years of teaching and research experience in soft computing and computer networks.

Dr. V. P. Singh is PhD and ME in Computer Science from Thapar University, Patiala, India. He is presently serving as assistant professor in the Computer Science and Engineering Department of Thapar University, India. His research interests include soft computing, Computer networks, Computer forensics and Cyber Law. He has many research publications in reputed Journals and Conferences.

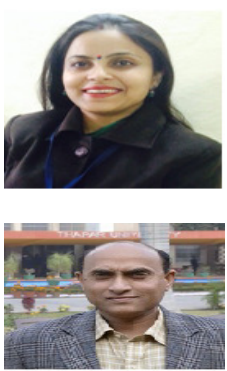

\section{APPENDIX I}

\section{Proposed Algorithm: FLCS}

\section{NOTATIONS}

FMM - Fuzzy Mixed Metric, MF- Membership Function, DS - Degree of Strength

\section{LSPs Setup System (LsS)}

(a) Computation of (FMM) to find ordered LSPs

Let MF and $\mathrm{Y}$ is any value from the defined regions

STEP 1 Put Y into MF system.

STEP 2 Check the area for both the variables $(L, D)$ that in which $Y$ is lying ie

\section{Linguistic term $\mathrm{L}$}

1) If perfectly lying in VL area, then assign the computed DS to the fuzzy set VL.

2) If in the area VL \& L, then compute the DS and assign to both fuzzy sets.

3) If perfectly lying in $\mathbf{L}$ area, then assign the computed DS to the fuzzy set $\mathbf{L}$.

4) If in the area $\mathbf{L} \boldsymbol{\&} \mathbf{M}$, then compute the DS and assign to both fuzzy sets.

5) If exactly in $\mathbf{M}$ area, then assign the computed DS to the fuzzy set $\mathbf{M}$. 
6) If in area $\mathbf{M} \boldsymbol{\&} \mathbf{H}$, then compute the DS and assign the same to both fuzzy sets.

7) If exactly in $\mathbf{H}$ area, then assign the computed DS to the fuzzy set $\mathrm{H}$.

8) If in area $\mathbf{H} \boldsymbol{\&} \mathbf{V H}$, then compute the DS and assign the same to both fuzzy sets.

9) If in area VH, then assign the computed DS to the fuzzy set VH.

\section{Linguistic term D}

1) If perfectly lying in $\mathbf{V L}$ area, then assign the computed DS to the fuzzy set $\mathbf{V L}$.

2) If in the area $\mathbf{V L} \boldsymbol{\&} \mathbf{L}$, then compute the DS and assign to both fuzzy sets.

3) If perfectly lying in $\mathbf{L}$ area, then assign the computed DS to the fuzzy set $\mathbf{L}$.

4) If in the area $\mathbf{L} \boldsymbol{\&} \mathbf{M}$, then compute the DS and assign to both fuzzy sets.

5) If exactly in $\mathbf{M}$ area, then assign the computed DS to the fuzzy set $\mathbf{M}$.

6) If in area $\mathbf{M} \boldsymbol{\&} \mathbf{H}$, then compute the DS and assign the same to both fuzzy sets.

7) If exactly in $\mathbf{H}$ area, then assign the computed DS to the fuzzy set $\mathrm{H}$.

8) If in area $\mathbf{H ~ \& ~ V H , ~ t h e n ~ c o m p u t e ~ t h e ~ D S ~ a n d ~ a s s i g n ~ t h e ~ s a m e ~ t o ~ b o t h ~ f u z z y ~ s e t s . ~}$

9) If in area VH, then assign the computed DS to the fuzzy set $\mathbf{V H}$.

STEP 3 Applying the DS within the rule base obtained output Linguistic term LsS_value

1) If perfectly lying in $\mathbf{Z}$ area, then assign the computed DS to the fuzzy set $\mathbf{Z}$.

2) If in the area $\mathbf{Z} \& \mathbf{T}$, then compute the DS and assign to both fuzzy sets.

3) If perfectly lying in $\mathbf{T}$ area, then compute the DS and assign to linguistic term $\mathbf{T}$.

4) If in the area $\mathbf{T} \boldsymbol{\&} \mathbf{V S}$, then compute the DS and assign to both fuzzy sets.

5) If in the area VS, then compute the DS and assign to linguistic term VS.

6) If in the area VS \& $\mathbf{S}$, then compute the DS and assign to both fuzzy sets.

7) If in $\mathbf{S}$ area, then assign the computed DS to the fuzzy set $\mathbf{S}$.

8) If in area $\mathbf{S} \boldsymbol{\&} \mathbf{B}$, then compute the DS and assign the same to both fuzzy sets.

7) If exactly in $\mathbf{B}$ area, then assign the computed DS to the fuzzy set $\mathbf{B}$.

8) If in area $\mathbf{B} \boldsymbol{\&} \mathbf{V B}$, then compute the DS and assign the same to both fuzzy sets.

9) If in area VB, then assign the computed DS to the fuzzy set VB.

10) If in area VB \& $\mathbf{H}$, then compute the DS and assign the same to both fuzzy sets.

11) If in area $\mathbf{H}$, then assign the computed DS to the fuzzy set $\mathbf{H}$.

STEP 4 Compute the value of each rule using AND/MIN operation.

STEP 5 Apply SUM compositions to achieve the final DS for the output variables.

STEP 6 Stop

(b) Formation of Routing Information Base (RIB) about Selection of LSPs for every node N in the network constructs an IB. The LsS_value of different paths recognizes the status of network. Computation of the Preferential order of the LSPs to arrange all the set of nodes/ paths using an appropriate sorting technique to obtain preferential order of LSPs.

\section{Traffic splitting System (TSS)}

(a) Computation of Fuzzy Mixed metric (FMM) to find requirement of no. of LSPs using TSS_value.

Let $\mathrm{MF}$ and $\mathrm{Y}$ is any value from the available regions

STEP 1 Put Y into MF system.

STEP 2 Check the area for both the variables (UR, LC) that in which Y is lying

\section{Linguistic term UR}

1) If perfectly lying in $\mathbf{Z}$ area, then assign the computed DS to the fuzzy set $\mathbf{Z}$.

2) If in the area $\mathbf{Z} \& \mathbf{T}$, then compute the DS and assign to both fuzzy sets.

3) If perfectly lying in $\mathbf{T}$ area, then compute the DS and assign to linguistic term $\mathbf{T}$.

4) If in the area $\mathbf{T} \& \mathbf{V S}$, then compute the DS and assign to both fuzzy sets.

5) If in the area VS, then compute the DS and assign to linguistic term VS.

6) If in the area VS \& S, then compute the DS and assign to both fuzzy sets.

7) If in $\mathbf{S}$ area, then assign the computed DS to the fuzzy set $\mathbf{S}$.

8) If in area $\mathbf{S} \& \mathbf{B}$, then compute the DS and assign the same to both fuzzy sets. 
7) If exactly in $\mathbf{B}$ area, then assign the computed DS to the fuzzy set $\mathbf{B}$.

8) If in area $\mathbf{B} \boldsymbol{\&} \mathbf{V B}$, then compute the DS and assign the same to both fuzzy sets.

9) If in area VB, then assign the computed DS to the fuzzy set VB.

10) If in area $\mathbf{V B} \boldsymbol{\&} \mathbf{H}$, then compute the DS and assign the same to both fuzzy sets.

11) If in area $\mathbf{H}$, then assign the computed DS to the fuzzy set $\mathbf{H}$.

Linguistic term $\mathbf{L C}$

1) If perfectly lying in VL area, then assign the computed DS to the fuzzy set VL.

2) If in the area VL \& $\mathbf{L}$, then compute the DS and assign to both fuzzy sets.

3) If perfectly lying in $\mathbf{L}$ area, then assign the computed DS to the fuzzy set $\mathbf{L}$.

4) If in the area $\mathbf{L} \& \mathbf{M}$, then compute the DS and assign to both fuzzy sets.

5) If exactly in $\mathbf{M}$ area, then assign the computed DS to the fuzzy set $\mathbf{M}$.

6) If in area $\mathbf{M} \boldsymbol{\&} \mathbf{H}$, then compute the DS and assign the same to both fuzzy sets.

7) If exactly in $\mathbf{H}$ area, then assign the computed DS to the fuzzy set $\mathrm{H}$.

8) If in area $\mathbf{H} \& \mathbf{V H}$, then compute the DS and assign the same to both fuzzy sets.

9) If in area VH, then assign the computed DS to the fuzzy set VH.

STEP 3 Applying the DS within the rule base obtained Output Linguistic term TSS_value

1) If perfectly lying in NC area, then assign the computed DS to the fuzzy set NC.

2) If in the area $\mathbf{N C} \& \mathbf{I O}$, then compute the DS and assign to both fuzzy sets.

3) If perfectly lying in IO area, then assign the computed DS to the fuzzy set IO.

4) If in the area IO $\& \mathbf{I W}$, then compute the DS and assign to both fuzzy sets.

5) If exactly in IW area, then assign the computed DS to the fuzzy set IW.

6) If in area IW \& IT, then compute the DS and assign the same to both fuzzy sets.

7) If exactly in IT area, then assign the computed DS to the fuzzy set IT.

8) If in area IT \& ALL, then compute the DS and assign the same to both fuzzy sets.

9) If in area ALL, then assign the computed DS to the fuzzy set ALL.

STEP 4 Compute the value of each rule using AND/MIN operation.

STEP 5 Apply SUM compositions to achieve the final DS for the output variables.

STEP 6 Stop

(b) Formation of Routing Information Base (RIB) consists of information of Traffic splitting for every LSP in the network. It contains information of status (utilization rate, link capacity) of paths. Traffic splitting among computed Fuzzy based LSPs is obtained according to the output response (TSS_value) received.

\section{APPENDIX II}

\section{Numerical of illustrative example with obtained results}

\section{LSPs Setup System (LsS)}

load $=\mathbf{8 5 8}$

$\mu_{\text {load }}=\left\{\mu_{\mathrm{VL}}, \mu_{\mathrm{L}}, \mu_{\mathrm{M}}, \mu_{\mathrm{H}}, \mu_{\mathrm{VH}}\right\}$ $=\{0,0,0, .62, .41\}$

delay $=.936$

$\mu_{\text {delay }}=\left\{\mu_{\mathrm{VL}}, \mu_{\mathrm{L}}, \mu_{\mathrm{M}}, \mu_{\mathrm{H}}, \mu_{\mathrm{VH}}\right\}$ $=\{0.33,0.17,0,0,0,0\}$

Activate rules, Substitute membership values \& applying MIN operation 
International Journal in Foundations of Computer Science \& Technology (IJFCST) Vol.6, No.2, March 2016

Table 8. Activated values

\begin{tabular}{|c|c|c|c|c|c|}
\hline D & VL & L & M & H & VH \\
L & .33 & .17 & & & \\
\hline VL & Z & T & VS & S & B \\
\hline L & T & VS & S & B & B \\
\hline M & T & VS & S & B & B \\
\hline H & VS & S & B & VB & VB \\
\hline $\mathbf{6 2}$ & .33 & .17 & & & \\
\hline VH & S & B & B & VB & H \\
.41 & .33 & .17 & & & \\
\hline
\end{tabular}

Composition is to combine the effects of all applicable rules and will give the best weighted influence to all firing rules.

$$
\begin{aligned}
& \mathrm{Z}=\text { Rule } 1=0 \\
& \mathrm{~T}=\text { Rules }(2+6+11)=0 \\
& \text { VS=Rules }(3+7+12+16)=0+0+0+.25=.33 \\
& \mathrm{~S}=\text { Rules }(4+8+13+17+21)=0+0+0+.17+.33=.50 \\
& \mathrm{~B}=\text { Rules }(5+9+10+14+15+18+22+23)=0+0+0+0+0+0+.12+0=.17 \\
& \text { VB=Rules }(19+20+24)=0 \\
& \mathrm{H}=\text { Rule } 25=0
\end{aligned}
$$

\section{LsS_value}

$\mu_{\text {LsS_value }}=\left\{\mu_{\mathrm{Z}}, \mu_{\mathrm{T}}, \mu_{\mathrm{VS}}, \mu_{\mathrm{S}}, \mu_{\mathrm{B},} \mu_{\mathrm{VB}}, \mu_{\mathrm{H}}\right\}$

$$
=\{0,0, .33, \mathbf{0 . 5 0}, \mathbf{0 . 1 7}, 0,0\}
$$

\section{Compute decision functions (Centroid Method) \& Fuzzified decisions}

Output Center points

$$
\begin{aligned}
& \mathrm{Z}_{\mathrm{C}}=0.0 \\
& \mathrm{~T}_{\mathrm{C}}=0.15 \\
& \mathrm{VS}_{\mathrm{C}}=0.35 \\
& \mathrm{~S}_{\mathrm{C}}=0.50 \\
& \mathrm{~B}_{\mathrm{C}}=0.65 \\
& \mathrm{VB}_{\mathrm{C}}=0.85 \\
& \mathrm{H}_{\mathrm{C}}=1.0 \\
& \text { Output }=\mathrm{Z}^{*} \mathrm{Z}_{\mathrm{C}}+\mathrm{T}^{*} \mathrm{~T}_{\mathrm{C}}+\mathrm{VS}^{*} \mathrm{VS}_{\mathrm{C}}+\mathrm{S}^{*} \mathrm{~S}_{\mathrm{C}}+\mathrm{B}^{*} \mathrm{~B}_{\mathrm{C}}+\mathrm{VB} * \mathrm{VB}_{\mathrm{C}}+\mathrm{H}^{*} \mathrm{H}_{\mathrm{C}}
\end{aligned}
$$$$
=0 * 0.0+0 * .15+.33 * .35+.50 * .50+.17 * .65+0 * .85+0 * 1.0
$$$$
=0.0+0.0+.1155+.25+.1105+0.0+0.0=.476
$$ 
International Journal in Foundations of Computer Science \& Technology (IJFCST) Vol.6, No.2, March 2016

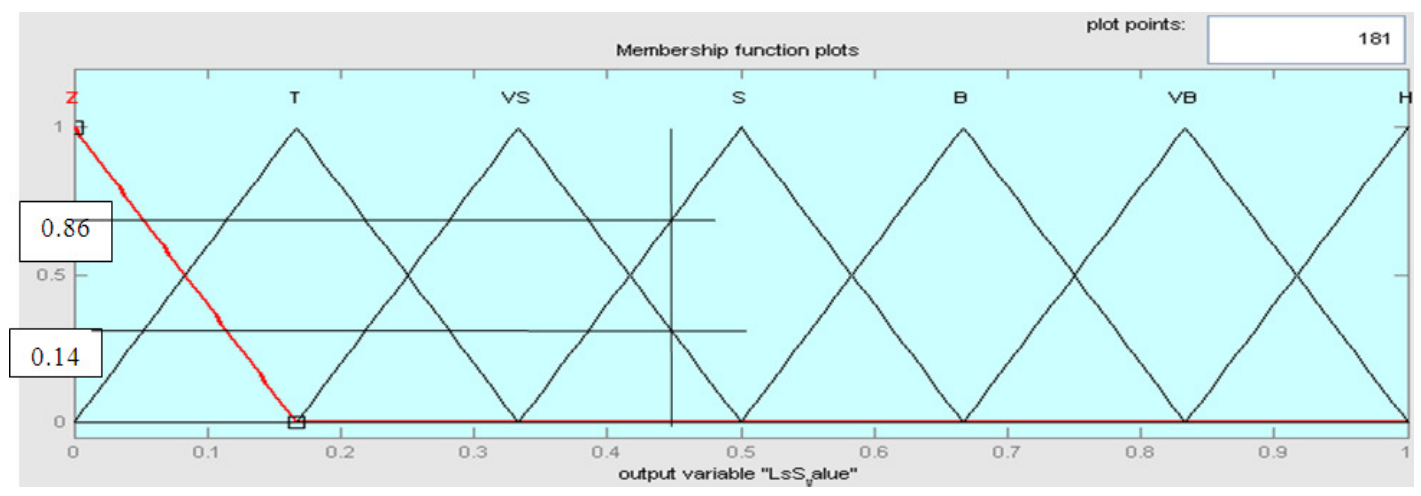

Figure 7. Fuzzified decision Output LsS_value indicates that link is $86 \%$ satisfactory and $14 \%$ good.

\section{Traffic splitting System (TSS)}

utilization_rate $=\mathbf{0 . 3 3}$

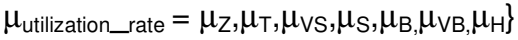

$$
=\{0,0,1,0,0,0,0\}
$$

link_capacity $=916$

$\mu_{\text {link_capacity }}=\left\{\mu_{\mathrm{VL}}, \mu_{\mathrm{L}}, \mu_{\mathrm{M}}, \mu_{\mathrm{H}}, \mu_{\mathrm{VH}}\right\}$ $=\{0,0,0,0.30,0.66\}$

\section{Activate rules, Substitute membership values \& applying Min operation}

Table 9. Activated rules

\begin{tabular}{|c|c|c|c|c|c|c|c|}
\hline UR & $\mathbf{Z}$ & $\mathbf{T}$ & VS & $\mathrm{S}$ & B & VB & $\mathrm{H}$ \\
\hline VL & $\mathrm{NC}$ & $\mathrm{NC}$ & $\mathrm{NC}$ & $\mathrm{NC}$ & IO & IO & IW \\
\hline $\mathrm{L}$ & $\mathrm{NC}$ & $\mathrm{NC}$ & $\mathrm{NC}$ & IO & IO & IW & IT \\
\hline $\mathrm{M}$ & $\mathrm{NC}$ & $\mathrm{NC}$ & IO & $\mathrm{IO}$ & IW & IW & IT \\
\hline $\begin{array}{l}\mathrm{H} \\
.30\end{array}$ & $\mathbf{N C}$ & IO & $\begin{array}{l}\text { IW } \\
.30\end{array}$ & IW & IT & IT & IK \\
\hline $\begin{array}{l}\text { VH } \\
.66\end{array}$ & IW & IW & $\begin{array}{l}\text { IT } \\
.66\end{array}$ & IT & IK & IK & IK \\
\hline
\end{tabular}

Composition is to combine the effects of all applicable rules and will give the best weighted influence to all firing rules.

$\mathrm{NC}=$ Rules $(1+2+3+4+8+9+10+15+16)=0+0+0+0+0+0+0+0=0$

$\mathrm{IO}=$ Rules $(5+6+11+12+17+18+23)=0+0+0+0+0+0+0+0=0$

IW=Rules $(7+13+19+24+25+29+30+20)=0+0+0+0.30+0+0+0+0+0=0.30$

IT $=$ Rules $(14+21+26+27+31+32)=0+0+0+0+0+0.66+0=0.66$

$\mathrm{A}=$ Rules $(28+33+34+35)=0+0+0+0+0+0+0=0$ 


\section{TSS_value}

$\mu_{\mathrm{TSS} \_ \text {value }}=\left\{\mu_{\mathrm{NC}}, \mu_{\mathrm{IO}}, \mu_{\mathrm{IW}}, \mu_{\mathrm{IT}}, \mu_{\mathrm{A}}\right\}$

$$
=\{0,0.30,0.66,0,0\}
$$

\section{Compute decision functions (Centroid Method) \& Fuzzified decisions}

Output Center points

$$
\begin{aligned}
& \mathrm{NC}_{C}=.1 \\
& \mathrm{IO}_{C}=.32 \\
& \mathrm{IW}_{\mathrm{C}}=.55 \\
& \mathrm{IT}_{\mathrm{C}}=.78 \\
& \mathrm{~A}_{\mathrm{C}}=1
\end{aligned}
$$
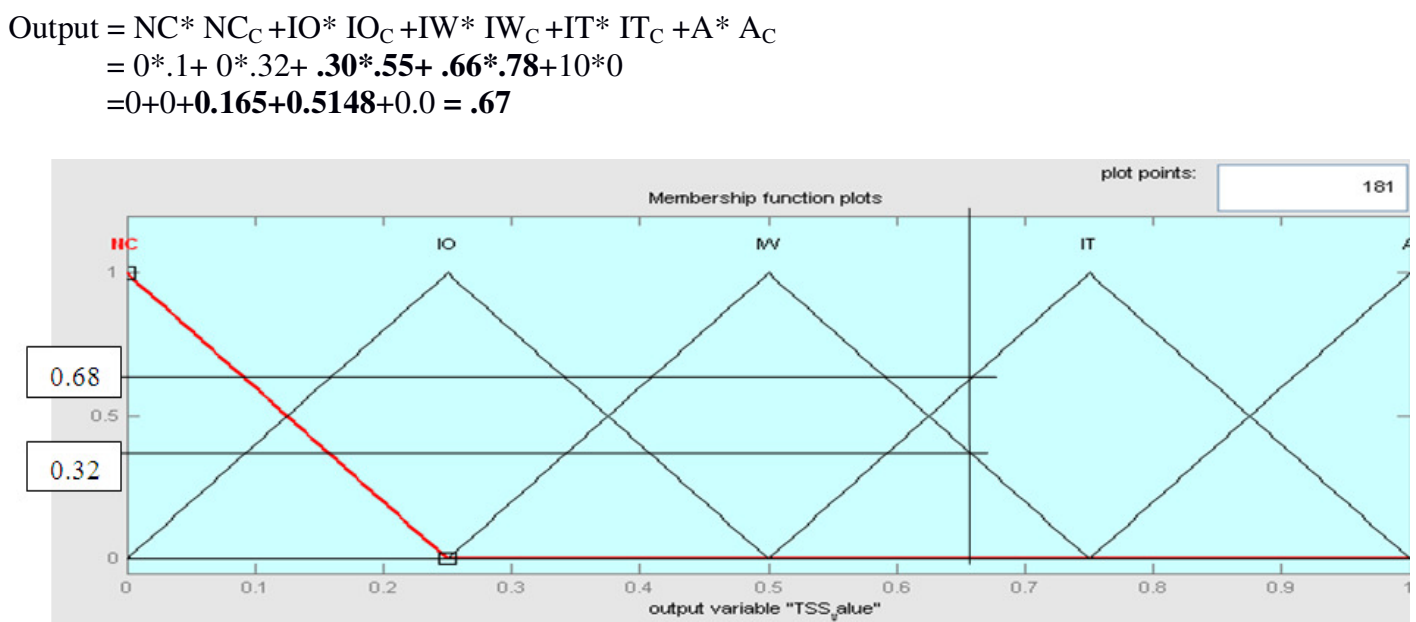

Figure 8. Fuzzified decision Output TSS_value indicates $68 \%$ increase of LSPs by 3 and $32 \%$ increases of LSPs by 2 are required. 